\title{
Neonatal ischemic liver failure or enterovirus hepatitis presenting as acute fulminant liver failure
}

\begin{abstract}
We report a case of neonatal acute hepatic failure due to enterovirus infection. The newborn presented with bradycardia, hypotonia and hypothermia in the first 5 days of life and later developed disseminated intravascular coagulation (DIC) and fulminant hepatitis.
\end{abstract}

Keywords: Acute liver failure, Haemophagocytic lymphohistiocytosis, Neonates, Serum transaminases
Case Report

Volume 10 Issue I - 2018

\author{
Viner Yuri, ${ }^{1,2}$ Hino Bayan, ${ }^{1,2}$ Arary Michael ${ }^{1,2}$ \\ 'Ziv Medical Center, Israel \\ ${ }^{2}$ Bar-llan University, Faculty of Medicine, Israel
}

Correspondence: Viner Yuri MD, Pediatric intensive care units Ziv Medical Center, Safed, Israel, Email viner0I@netvision.net.il

Received: February 09, 2018 | Published: February 23, 2018

\section{Introduction}

Neonatal acute liver failure (ALF) is a rare condition that carries a high mortality $(70 \%)$ without liver transplantation. ${ }^{1}$

In this presentation, we have attempted to identify clinical and laboratory features which may assist in optimal management of enterovirus-induced neonatal ALF. The exceptional feature of this case is the multidisciplinary approach employed for diagnosis and treatment, which lead to recovery from ALF without liver transplantation.

No cases of fulminant neonatal hepatic failure associated with very severe coagulopathy (prolonged prothrombin time (PT) and international normalized ratio (INR) and factor $\mathrm{V}$ levels $<4 \%$ ) which survived without liver transplantation, have been previously reported.

\section{Case Presentation}

A five day-old male presented to our emergency room with lethargy, weakness and poor appetite. A yellowish skin color, which had been absent at birth, was noted. The patient was born at our hospital by vaginal delivery after a full-term, uncomplicated gestation. The mother had received prenatal care; she had no history of sexually transmitted infections, and prenatal screening tests were negative. Meconium was present at delivery. The patient's birth weight was $3.1 \mathrm{~kg}\left(50^{\text {th }}\right.$ percentile $)$ and the 1-minute and 5-minute Apgar scores were 9 and 10, respectively. Breast-feeding was initiated. He was discharged home at 48 hours of age. His post-partum period was unremarkable apart from mild sleepiness. There was no history of the child vomiting, diarrhea or fever. The only medications he received were a recombinant DNA hepatitis B virus vaccine, a prophylactic vitamin $\mathrm{K}$ injection and antibacterial eye cream. He was discharged from the maternity ward with a serum bilirubin of $8 \mathrm{mg} / \mathrm{dL}$ and hematocrit 57\%, weight loss $6 \%$ from delivery.

He lived with his mother, father and two his healthy older sisters. The parents are non-consanguineous, the mother is a carrier of the gene for spinal muscular atrophy; the father is not. Shortly before giving birth the mother developed diarrhea and mild fever. On postpartum day 3 , the mother experienced severe abdominal pain. She visited the emergency department with her newborn and was discharged with no specific diagnosis, after a normal physical examination, complete blood count (CBC) and liver function tests (LFTs) that were without obvious pathology.

\section{Physical examination, vital signs and initial tests}

Upon examination, the child appeared weak and apathetic. Pulse rate was 199 beats per minute (bpm), peripheral capillary oxygen saturation $98 \%$ in room air, rectal temperature was $35.5^{\circ} \mathrm{C}$ and brachial blood pressure was 65/30. Capillary refill time (CRT) was slow at 5 seconds. Respiratory rate was 30 breaths per minute.

Weight was $2.7 \mathrm{Kg}$. (13\% less than birth weight). There was no spontaneous limb movement; he was hypotonic with a weak Moro reflex. The fontanelle was normotensive; pupils were round, even and responsive to light; skin was pale and yellow tinged. The abdomen was distended, soft, and tympanic, with normal bowel sounds. There was marked hepatomegaly. His stools were blood. His extremities were cool. The rest of the physical examination was unremarkable.

Laboratory evaluation revealed blood glucose $44 \mathrm{mg} / \mathrm{dL}$; total serum bilirubin $14.3 \mathrm{mg} / \mathrm{dL}$ (indirect bilirubin $13.3 \mathrm{mg} / \mathrm{dL}$ ), CBC normal hemoglobin and leukocytes but platelet count was 13,000 $\mathrm{mm}^{-3}$. Urinalysis revealed clear orange urine, with a specific gravity $<1.030, \mathrm{pH} 6.5$, nitrites negative, $1+$ urobilinogen, $3+$ bilirubin, $3+$ blood, protein negative, trace white cells and ketones.

Abdominal X-ray showed no evidence of obstruction or intestinal perforation.

\section{Initial treatment and response}

The patient was admitted to the pediatric intensive care unit (PICU). The initial management problems were encephalopathy, abdominal distention, bloody diarrhea and cardiovascular dysfunction, hypoglycemia and thrombocytopenia. Consensus criteria for severe sepsis with probable shock were present. The history suggested hypovolemia, due to diarrhea and poor oral intake. He was rapidly given intravenous (IV) fluids $0.9 \%$ saline in boluses of $10 \mathrm{ml} / \mathrm{kg}$ of body weight over a period of 5 to 10 minutes, and repeated as long as signs and symptoms of shock persisted. After a total volume of 60 
$\mathrm{ml} / \mathrm{kg}$, the infant's condition improved and thereafter blood pressure remained normal; there was no evidence of heart failure or volume overload. In addition, he was given IV fluids containing $25 \%$ glucose.

Core body temperature was controlled using both passive and active external rewarming. Blood glucose concentration stabilized and the patient rapidly became more responsive. CRT improved to 3 seconds; pulse dropped to $95 \mathrm{bpm}$ and oxygen saturation was $98 \%$.

Blood, urine and stool samples were sent for bacterial culture but lumbar puncture was not performed due to the severe thrombocytopenia. Treatment with intravenous ampicillin, cefotaxime and acyclovir was begun. Because of severe thrombocytopenia, hematuria and hematochezia, irradiated platelet concentrates and -immunoglobulin (IVIG) were administered. Assessment of coagulation function revealed severe coagulopathy with the PT activity decreased to below $10 \%$ of normal; INR was $>7$; fibrinogen $1200 \mathrm{mg} / \mathrm{dL}$; D-dimer 5.87 $\mu \mathrm{g} / \mathrm{ml}$, activated partial thromboplastin time (aPTT) $>110$ seconds; factor VIII 66\% (normal).

On the next day, cofactor $\mathrm{V}$ activity was $<4 \%$ of normal, or PT activity remained $<10 \%$ of normal, or the INR was $>15$, fibrinogen $<100 \mathrm{mg} / \mathrm{dL}$ and D-dimer was $3.87 \mu \mathrm{g} / \mathrm{ml}$. PTT $>110$ seconds, factor VIII $21 \%$. Schistocytes were seen on peripheral blood smear. Thrombocyte count was $<10000 \mathrm{~mm}^{-3}$.

The patient remained stable hemodynamically and respiratory but continued to be encephalopathic. The fontanelle was consistently normotensive; stools remained bloody and profuse but the abdominal distention decreased. Clinical jaundice was unchanged.

At this stage the differential diagnosis included ischemic liver injury secondary to shock, hepatitis or less likely metabolic liver disease. Management included a multidisciplinary approach and prompted the need for early consultation with a liver transplantation center. However, since neonatal liver transplantations was at this time unavailable in Israel, conservative management was the only practicable option.

Further laboratory studies are summarized in table 2. Genera work-up included: serum electrolytes $\left(\mathrm{Na}^{+}, \mathrm{K}^{+}, \mathrm{Cl}^{-}, \mathrm{PO}_{4}^{-}, \mathrm{Mg}^{2+}\right.$, $\mathrm{Ca}^{2+}$, blood urea nitrogen(BUN), creatinine, lactate dehydrogenase (LDH), lactate, ammonia, ferritin, blood gas analysis; CBC; Coomb's test; blood and urine cultures; blood group determination, aspartate transaminase (AST), alanine transaminase (ALT), alkaline phosphatase (ALP), $\gamma$-glutaryl transferase (GGT), total and conjugated bilirubin, $\alpha$-fetoprotein.

We continued additional investigations that included:

Metabolic work-up: plasma tyrosine, phenylalanine, methionine, $\alpha$-fetoprotein and urine succinyl acetone.

Red cell galactose-1-phosphate uridyl transferase activity.

Ferritin.

Blood lactate.

Plasma amino acids, ammonium and urinary organic acid.

Transferrin isoelectric focusing.

Plasma very long chain fatty acid (VLCFA).

Infectious work-up: polymerase chain reaction (PCR) of blood for Hepatitis A and B; human herpes virus (HHV)-1, 2 and 6; cytomegalovirus (CMV); Epstein-Barr virus (EBV); varicella zoster virus(VZV); echovirus; adenovirus; enterovirus; parvovirus B19; paramyxovirus; Venereal Disease Research Laboratory (VDRL) test (maternal); cultures of blood, urine and stool.

Other: Blood triglycerides, cholesterol,

Serum $\alpha$-1-antitrypsin level

\section{Echocardiography for vascular/hypoxic-ischemic pathology}

Several imaging studies were performed, including abdominal sonographies, which demonstrated the liver to be $48 \mathrm{~mm}$ in size, with normal structure and texture and no focal findings. The portal vein was $4 \mathrm{~mm}$ in diameter and the ductus venous was patent. The gallbladder was partially contracted, with no apparent cholelithiasis. The spleen was slightly enlarged, measuring up to $60 \mathrm{~mm}$, with normal structure and texture. Other findings were normal renal and adrenal parenchyma, small-volume peritoneal and pleural effusions. Abdominal X-ray showed no evidence of obstruction or intestinal rupture. Cranial ultrasound (US) revealed no calcifications or evidence of bleeding. Echocardiography was normal.

\section{Management rationale}

In our case, such an early presentation with liver failure (on postpartum day 5) increased the likelihood of conditions such as congenital infections, neonatal hemochromatosis, hemophagocytic syndrome, congenital leukemia, inborn errors of metabolism or an hypoxic ischemic insult. The birth history was unremarkable with good Apgar scores, so hypoxic ischemic insult to the liver was unlikely. Inborn errors of metabolism may in theory present on the third day of life, but his clinical presentation, together with the negative initial metabolic evaluation were less suggestive of an inborn error. A normal serum alpha-1-antitrypsin level excluded alpha-1antitrypsin deficiency.

Neonatal hemochromatosis $(\mathrm{NH})$ and hemophagocytic lymphohistiocytosis (HLH) were two possible diagnoses with the severe liver failure soon after birth. However, NH usually presents as acute decompensated end-stage liver disease with a small liver and often normal serum transaminases (TAs); whereas in our case, there was marked hepatomegaly and increase in serum TAs. The absence of fever, cytopenias, hyperferritinemia and hypertriglyceridaemia excluded the diagnosis of HLH. Liver and lip or salivary gland biopsy for any extrahepatic iron deposits, and bone-marrow examination were not performed due to the patient's coagulopathy and unstable clinical condition.

Real-time PCR (RT-PCR) and culture of a throat swab sample were positive for enterovirus. PCR for HSV-1\&2 and CMV was negative. Antibiotic therapy and acyclovir were discontinued.

The clinical features of hypothermia, hepatosplenomegaly, thrombocytopenia, severe coagulopathy, encephalopathy and acute hepatitis together with a positive PCR and culture for enterovirus, together with a history of a "viral infection" in the mother, lead us to diagnose neonatal enterovirus infection, presenting as acute fulminant liver failure. The principles of management in such a case include minimizing complications as far as possible and avoiding the administration of drugs that have no proven beneficial effect. 
Encephalopathy: We did not use any conventional grading system for encephalopathy that usually is used for adults and older children. We elected not to carry out measurements of intracranial pressure (ICP) Evaluation of encephalopathy was based on the Glasgow coma scale (GCS), reaction and size of pupils, signs of the Cushing reflex (irregular breathing pattern, presence of bradycardia and hypertension with a wide pulse pressure), change of body posture (decorticate or decerebration) and evaluation of anterior fontanelle tension. The initial presentation was inconsolable crying and later this progressed to somnolence. Initially GCS was 10-11 falling to 9-10 over 48 hours. The pupils reacted well to light and no seizures were seen. Bradycardia ( $80 \mathrm{bpm}$ ) was noted for 2 days. The baby was nursed in a quiet environment with as little stimulation as possible to minimize acute increases in intra-cranial pressure. Serum sodium levels were maintained in normal range.

Sedation was avoided in order to avoid interference with neurological status. Mannitol, lactulose and exchange transfusion were not used. A protein intake of $1.5 \mathrm{gram} / \mathrm{kg}$ was provided via NG feeding without complication.

The baby became alert after 7 days, GCS normalized, the fontanelle became normotensive, and movements and reflexes became normal.

Infection control: The baby was initially treated with cefotaxime, ampicillin and acyclovir. The work up for infections: from all investigations were done the real-time polymerase chain reaction (RT-PCR) and culture of a throat swab sample came back positive for enterovirus. PCR for HSV-1\&2 was negative. The blood was negative for cytomegalovirus PCR and antigen test. The blood and urine cultures were negative for bacterium. Antibiotic therapy and acyclovir were discontinued.

On the $10^{\text {th }}$ day, increasing apathy was noted accompanied by a markedly increased absolute leukocyte count and pyrexia. Ampicillin and Gentamicin were administered and urine culture was positive for ESBL Escherichia coli $(100,000 \mathrm{CFU} / \mathrm{ml}$. Later Amikacin was successfully substituted.

Coagulopathy: In coagulation assessment had be noticed severe coagulopathy: cofactor $\mathrm{V}$ activity decreases below $4 \%$ of normal, or if the prothrombin time activity decreases to below $10 \%$ of normal, or the INR is greater than 15 ,fibrinogen less 100 and D-diamer was 5.87 . PTT more $110 \mathrm{sec}$ and severe thrombocytopenia. Factor VIII also was low. The differential diagnosis of the child's coagulopathy was acute hepatic failure with fibrinolysis due to ischemic liver injury secondary to shock and/or hepatitis, or intravascular coagulation (DIC).

Bleeding was minimized by minimal handling, IV platelet concentrates (thrombocyte maintained over $15000 \mathrm{~mm}^{-3}$ ).and regular infusions of fresh frozen plasma (FFP) $(10-15 \mathrm{~mL} / \mathrm{kg})$. Since coagulation failed to improve deranged, we moved on to a continuous infusion of FFP was infused in a larger dose continuously $(2-3 \mathrm{ml} / \mathrm{kg}$ / $\mathrm{hr})$. Cryoprecipitate antihemophilic factor was added when fibrinogen levels fell $<100 \mathrm{mg} / \mathrm{dL}$.

Fat-soluble vitamins of the ADEK group and additional IV vitamin $\mathrm{K}$ ( $1 \mathrm{mg}$ q. 3 days) were administered.

The baby had received cimetidine to prevent gastric bleeding. Prothrombin complex concentrates and rhFVIIa for events were not required.
After 10 days: Factor V was $21 \%$ of normal and $66 \%$ two weeks later. INR normalized after one month. Fibrinogen normalized after 10 days. The platelet count increased to $35,000 \mathrm{~mm}^{-3}$ after 10 days. No clinical bleeding occurred.

One-packed red cells transfusion was administered a when blood hemoglobin concentration decreased to $6 \mathrm{mg} / \mathrm{dL}$. Red cells were sparingly given so as not to exacerbate the already-significant jaundice.

Hemodynamic: The early hemodynamic changes in the baby were similar to those seen in the systemic inflammatory response syndrome (SIRS), including bradycardia, with decreased systemic peripheral vascular resistance and normal cardiac output on echocardiography. Improved capillary refill time, alertness and diuresis were achieved without inotropes. Later generalized edema developed, most probably due to capillary leak and hypoalbuminemia, which resolved without the use of diuretics nor prostacycline and $\mathrm{N}$-acetylcysteine.

Metabolic derangement and liver function: Initially elevated liver enzymes (ALT and AST 12000 unit's level) severe coagulopathy, hypoalbuminemia and indirect hyperbilirubinemia were noted. Subsequently, these enzymes decreased markedly, while the coagulopathy worsened. Direct hyperbilirubinemia developed, with increased ALP and GGT.

Doppler ultrasound of the liver vasculature showed the intra- and extra-hepatic bile ducts to be non-dilated. Blood flow through the portal vein was hepatopetal and unobstructed, up to and including the hepatic veins. The blood vessels around the patent ductus venosus in the left lobe were dilated and some free peritoneal fluid was seen. Due to an increase in the direct fraction of serum bilirubin, without an equivalent increase in the indirect fraction, ursodeoxycholic acid (35 $\mathrm{mg} \mathrm{X}$ 3/day po.) was added to the treatment.

Hypoglycemia was present in the baby. The classic signs and symptoms of hypoglycemia were masked due to hepatic encephalopathy, hence regular blood glucose monitoring was mandatory. Continuous enteral feeding via NG tube was initiated using a commercial lactose-free, hypoallergenic milk casein hydrolysate solution containing 55\% medium-chain triglycerides, docosahexaenoic acid and arachidonic acid. The blood glucose concentration was labile and resulted in hypoglycemic events, which were not always orally correctable and these required IV $25 \%$ glucose boluses. Protein intake was $1.0-1.5 \mathrm{~g} / \mathrm{kg} / \mathrm{day}$. Acid-base imbalance and lactic acidosis were not seen. Hypokalemia was corrected using a potassium solution, administered enterally.

Ammonia levels and encephalopathy were not correlated.

Renal complications: Urine output and renal function were normal, and there was no evidence of hepatorenal syndrome. On sonography, his kidneys had a network of echogenic foci in the pyramids, probably consistent with acute tubular necrosis (ATN).

During the second week, the patient's vital signs and clinical condition gradually stabilized. On the $12^{\text {th }}$ day of hospitalization, the patient gradually became more responsive and a significant improvement in his overall condition was apparent, with a marked improvement in liver enzymes and an improving coagulation profile. Albumin rose to $3.5 \mathrm{~g} / \mathrm{dL}$ with resolution of ascites and peripheral edema, while urine output and renal function remained normal. 
He was transferred from the PICU to the pediatric department. The feeding regimen was switched to intermittent boluses with a baseline continuous NG feeding in a rate of $10 \mathrm{ml} / \mathrm{hr}$. Breastfeeding was encouraged on demand and the patient began to gain weight, up to a final discharge weight of $3.4 \mathrm{~kg}$. The hypoglycemic events became less frequent, but prolonged fasting still resulted in hypoglycemia. Repeat searches for enterovirus in stool and throat were negative.

The patient was discharged at the age of 21 days in good overall condition, with normal vital signs and stable LFTs. He was prescribed oral ADEK vitamins and ursodeoxycholic acid (35 mg x 3/day). His progress is currently being followed periodically in an outpatient setting. The patient is well, with normal growth but still has mildly elevated GGT: $114 \mathrm{IU} / \mathrm{L}$ (normal for age is 1-55 IU/L) 3 months after discharge.

\section{Discussion}

This 5-day-old boy presented with signs of severe sepsis. His condition met the consensus definition of severe sepsis. ${ }^{2}$ Neonates with sepsis may present in, or progress to septic shock, exemplified initially by cardiovascular dysfunction requiring fluid resuscitation or inotropic support. ${ }^{3}$ Severe sepsis with shock is associated with an imbalance between oxygen delivery and demand. The treatment strategy to correct this imbalance is referred to as early goal-directed therapy using aggressive fluid administration (usually up to $60 \mathrm{cc} / \mathrm{kg}$ ) with concomitant assessment of response. Lack of response would be an indication for vasopressor therapy ${ }^{4-7}$ In this case, the patient had a good clinical response to fluid therapy and vasopressors were unnecessary. Suggestions for cardiovascular therapeutic end points include a capillary refill time of $<2$ seconds, normal pulses with no difference between peripheral and central pulses, warm extremities, urine output $>1 \mathrm{ml} / \mathrm{kg} / \mathrm{h}$, low serum lactate.

In the absence of widely available or well-tested methods for quantifying hemodynamic compromise in septic shock in neonates, clinicians generally rely on vital signs and physical examination to make decisions about therapy. Although mean arterial pressure (MAP) may not reflect systemic blood flow, monitoring blood pressure and other measures such as capillary refill time and urine output provide indirect information on the adequacy of organ blood flow. ${ }^{5,6}$

The blood concentration of hemoglobin was $10 \mathrm{mg} / \mathrm{dL}$ and there was no need for blood transfusions. We were not able to predict the development of fulminant hepatic failure (FHF). From a retrospective point of view, we cannot was have prompted the administration of prostacycline and $\mathrm{N}$-acetylcysteine which have been found to be beneficial for oxygen metabolism. 8,9

Several pathogens have been associated with sepsis in the neonatal period. The predominant agents are bacterial, but viruses, including herpes simplex and enteroviruses, have been associated with fulminant neonatal hepatitis with multi-organ involvement and hepatic necrosis with high mortality. ${ }^{10-12}$ Echovirus, particularly serotype 11, is the most frequently identified virus. It is postulated that echovirus causes hepatic failure by vascular, rather than by direct hepatocyte damage, ${ }^{13}$ that were returned us to using prostacycline and $\mathrm{N}$-acetylcysteine in treatment fulminant neonatal hepatitis due to enterovirus.

Outcomes range from spontaneous recovery to a rapidly fatal fulminant course. Mortality has been estimated to be as high as $80 \% .{ }^{14}$
The role of the oral antiviral agent pleconaril in neonatal infection is currently being evaluated in a multicenter study but there is as yet insufficient evidence of its efficacy in enterovirus infection. ${ }^{15-17}$

The definition of ALF in children and neonates is controversial. The accepted adult criteria ALF is defined as a ${ }^{18}$ are unsatisfactory for infants. It is very difficult to identify signs of encephalopathy in this age group; and it can be a very late event in the course of the disease. Bhaduri and Mieli-Vergani have addressed this issue and proposed the following definition of ALF in children: rare multisystem disorder in which severe impairment of liver function, with or without encephalopathy, occurs in association with hepatocellular necrosis in a patient with no recognized underlying chronic liver disease. ${ }^{19}$

The Pediatric Acute Liver Failure Study Group (PALFSG) defined the diagnosis of ALF in pediatric patients; in neonates as the presence of biochemical evidence of acute hepatic injury and an INR $>1.5$ with encephalopathy or INR $>2$ without encephalopathy. ${ }^{20}$ The presence of a multisystem disorder allowed us to confidently define the patient's condition as FHF.

FHF can be schematically classified into seven categories: metabolic, infective, toxic, autoimmune, malignancy-induced, vascular-induced, and undetermined. In infants, metabolic disease is the most frequent cause of FHF. Whatever the age, undetermined FHF is frequently observed. ${ }^{21-24}$

In our case, such an early presentation for liver failure on day 5 pointed to conditions like infections, neonatal haemochromatosis, haemophagocytic syndrome, congenital leukemia or hypoxic ischemic insult. The birth history was unremarkable with good Apgar scores, so hypoxic ischemic insult to the liver was unlikely. Inborn errors of metabolism generally present on day 5 of life and they usually present with cholestasis and not high liver enzymes. The presentation together with the negative urine reducing substances initially did not suggest diagnoses like galactosaemia, hereditary fructose intolerance or urea cycle defect. The negative urine nitrosonaphthanol test and succinylacetate for thrice also ruled out tyrosinaemia type 1. Mitochondrial diseases, fatty acid oxidation defects were unlikely with the normal serum lactate, carnitine, acylcarnitine pattern and urine organic acid pattern. Peroxisomal disorder was again unlikely with the normal blood very long chain fatty acid assay. Serum alpha-1-antitrypsin level was normal that ruled out alpha 1-antitrypsin deficiency. Neonatal haemochromatosis $(\mathrm{NH})$ and haemophagocytic lymphohistiocytosis (HLH) were two highly probable diagnoses, but there were absence the high serum ferritin level and hypertriglyceridaemia. However, NH usually presents as acute decompensated end stage liver disease with small liver and often normal serum transaminases; whereas in our case, there was marked hepatomegaly and increase in serum transaminases.

The clinical features of hypothermia, hepatosplenomegaly, thrombocytopenia, severe coagulopathy, encephalopathy and acute hepatitis together with a positive PCR and culture for enterovirus, together with a history of a "viral infection" in the mother, lead us to diagnose neonatal enterovirus infection, presenting as acute fulminant liver failure.

The liver has a dual blood supply and receives blood from both the hepatic artery and portal vein. These blood supplies mix within hepatic sinusoids and subsequently drain through multiple hepatic veins. ${ }^{25}$ 
Blood flow through the liver is therefore somewhat protected from the acute and chronic changes in cardiac output and systemic blood pressure. However, severe acute and/or prolonged changes in cardiac function may lead to hepatic dysfunction. ${ }^{26}$ Ischemic hepatitis refers to the disorders that cause liver injury by reducing oxygen delivery to the liver. ${ }^{27}$ This is most commonly seen in the setting of severe hypotension and global hepatic hypoperfusion. The clinical hallmark of ischemic hepatitis consists of an abrupt elevation in the levels of serum liver ATs, (AST and ALT), and lactate dehydrogenase (LDH), which peak within 1 to 3 days of an episode of severe hypotension. Shock liver syndrome (SLS), is a well-recognized complication of reduced hepatic blood flow in low cardiac output states. ${ }^{28,29}$ Our patient had a documented episode of hemodynamic instability prior to the onset of hepatitis. There was a marked rise in ALT, AST and LDH levels in the first $96 \mathrm{~h}$ after admission, with a rapid decline to near-normal levels within 14 days. Serum bilirubin levels also rose, but not to the extent of transaminase levels. Ischemic hepatitis can occur during illnesses associated with diminished hepatic blood flow. The possibility that the observed abrupt rise in serum liver AT levels in a patient with presumed ischemic hepatitis may actually result from an acute viral or non-viral hepatitis should always be considered. However, this rapid rise and fall in AT levels is unusual in acute viral hepatitis in which the transaminase levels return to normal over weeks rather than days. ${ }^{27-29}$

After birth, the ductus venosus gradually closes. In the event of perinatal hypoxemia and circulatory failure, the ductus venosus may remain patent, leading to a significant reduction in blood-flow to the right liver, compared with the left liver. ${ }^{30-32}$

This consideration has raised the possibility that the liver might be more susceptible to hypoxia and ischemia in the infant with septic shock (although we did not see a difference in blood-flow on US between the left and right lobe of the liver)

\section{Conclusion}

Rapid determination of the underlying cause for the FHF and providing a diagnosis within a short time-frame is essential because instituting specific therapy and determining the potential for liver regeneration has an impact on the need for liver transplantation. We know that early referral and closer follow-up is necessary for timely admission to liver transplant centers to enable screening and proper preparation of these patients for liver transplantation. In our case, we had no choice but to conclude that liver transplantation remains the therapeutic choice for fulminant hepatic failure in this infant. We think that one is the most important point in our case. This situation may occur in many clinics worldwide, in which liver transplantation is not feasible.

In addition to lack of access to liver transplantation, the procedure can be unavailable due to medical contraindications. ${ }^{23,33,34}$ It is contraindicated in patients with advanced complications of ALF, such as fixed and dilated pupils, uncontrolled sepsis, and severe respiratory failure (adult respiratory distress syndrome) or conditions not treatable by liver replacement, such as mitochondrial cytopathies with neurological involvement, HLH, giant-cell hepatitis with Coombs-positive hemolytic anemia (which recurs after transplant) and malignancies. Though worse than for older children, the outcome of neonatal liver transplantation is acceptable, with a patient survival of about $65 \%$. $^{1,23,33,34}$

\section{Acknowledgments}

None.

\section{Conflict of interest}

None.

\section{References}

1. Devictor D, Tissieres P. Pediatric liver transplantation: where do we stand? Where we are going to? Expert Rev Gastroenterol Hepatol. 2013;7(7):629-641.

2. Goldstein B, Giroir B, Randolph A. International pediatric sepsis consensus conference: definitions for sepsis and organ dysfunction in pediatrics. Pediatr Crit Care Med. 2005;6(1):2-8.

3. Haque KN. Defining common infections in children and neonates. $J$ Hosp Infect. 2007;65 Suppl 2:110-114.

4. De Oliveira CF. Early goal-directed therapy in treatment of pediatric septic shock. Shock. 2010;34 Suppl 1:44-47.

5. Kissoon N, Orr RA, Carcillo JA. Updated American College of Critical Care Medicine--pediatric advanced life support guidelines for management of pediatric and neonatal septic shock: relevance to the emergency care clinician. Pediatr Emerg Care. 2010;26(11):867-869.

6. Brierley J, Carcillo JA, Choong K, et al. Clinical practice parameters for hemodynamic support of pediatric and neonatal septic shock: 2007 update from the American College of Critical Care Medicine. Crit Care Med. 2009;37(2):666-688

7. Parker MM, Hazelzet JA, Carcillo JA. Pediatric considerations. Crit Care Med. 2004;32(11 Suppl):S591-594.

8. Harrison PM, Keays R, Bray GP, et al. Improved outcome of paracetamol-induced fulminant hepatic failure by late administration of acetylcysteine. Lancet. 1990;335(8705):1572-1573.

9. O’Grady JG. Acute liver failure. In: O'Grady JG, Lake JR, Howdle PD, editors. Comprehensive Clinical Hepatology. London: Mosby; 2000. p. 30.1-30.20.

10. Verboon-Maciolek MA, Krediet TG, Gerards LJ, et al. Clinical and epidemiologic characteristics of viral infections in a neonatal intensive care unit during a 12-year period. Pediatr Infect Dis J. 2005;24(10):901904.

11. Verboon-Maciolek MA, Krediet TG, Gerards LJ, et al. Severe neonatal parechovirus infection and similarity with enterovirus infection. Pediatr Infect Dis J. 2008;27(3):241-245.

12. Kawada J, Kimura H, Ito Y, et al. Evaluation of systemic inflammatory responses in neonates with herpes simplex virus infection. $J$ Infect Dis 2004;190(3):494-498.

13. Wang J, Atchison RW, Walpusk J, et al. Echovirus hepatic failure in infancy: report of four cases with speculation on the pathogenesis. Pediatr Dev Pathol. 4(5):454-460.

14. Modlin JF. Perinatal echovirus infection: insights from a literature review of 61 cases of serious infection and 16 outbreaks in nurseries. Rev Infect Dis. 8(6):918-26.

15. Sawyer MH. Enterovirus infections: diagnosis and treatment. Semin Pediatr Infect Dis. 2002;13(1):40-47.

16. Aradottir E, Alonso EM, Shulman ST. Severe neonatal enteroviral hepatitis treated with pleconaril. Pediatr Infect Dis J. 2001;20(4):457459 
17. Abzug MJ. The enteroviruses: problems in need of treatments. J Infect. 2014;68 Suppl 1:S108-114.

18. Trey C, Davidson CS. The management of fulminant hepatic failure. Prog Liver Dis. 1970;3:282-398.

19. Bhaduri BR, Mieli-Vergani G. Fulminant hepatic failure: pediatric aspects. Semin Liver Dis. 1996;16(4):349-355.

20. Squires R h. J, Shneider B 1., Bucuvalas J, et al. Acute Liver Failure In Children: The First 348 Patients In The Pediatric Acute Liver Failure Study Group. J Pediatr 2006;148:652-8.

21. Centeno MA, Bes DF, Sasbón JS. Mortality risk factors of a pediatric population with fulminant hepatic failure undergoing orthotopic liver transplantation in a pediatric intensive care unit. Pediatr Crit Care Med. 2002;3(3):227-233.

22. Chen H-L, Chang C-J, Kong M-S, et al. Pediatric fulminant hepatic failure in endemic areas of hepatitis B infection: 15 years after universal hepatitis B vaccination. Hepatology. 2004;39(1):58-63.

23. Devictor D, Desplanques L, Debray D, et al. Emergency liver transplantation for fulminant liver failure in infants and children Hepatology. 1992;16(5):1156-1162.

24. Durand P, Debray D, Mandel R, et al. Acute liver failure in infancy: a 14-year experience of a pediatric liver transplantation center. $J$ Pediatr 2001;139(6):871-876.

25. Lautt WW, Greenway CV. Conceptual review of the hepatic vascular bed. Hepatology. 7(5):952-963.
26. Sherlock $\mathrm{S}$. The liver in heart failure; relation of anatomical, functional, and circulatory changes. Br Heart J. 1951;13(3):273-293.

27. Bynum TE, Boitnott JK, Maddrey WC. Ischemic hepatitis. Dig Dis Sci. 1979;24(2):129-135.

28. Jacquemin E, Saliba E, Blond $\mathrm{MH}$, et al. Liver dysfunction and acute cardiocirculatory failure in children. Eur J Pediatr 1992;151(10):731734.

29. Garland JS, Werlin SL, Rice TB. Ischemic hepatitis in children: diagnosis and clinical course. Crit Care Med. 1988;16(12):1209-1212.

30. Rudolph AM. Hepatic and Ductus Venosus Blood Flows During Fetal Life. Hepatology. 2007;3(2):254-258.

31. Itskovitz J, Goetzman BW, Rudolph AM. Effects of hemorrhage on umbilical venous return and oxygen delivery in fetal lambs. $A m J$ Physiol. 1982;242(4):H543-548.

32. Rudolph AM, Heymann MA. Circulatory changes during growth in the fetal lamb. Circ Res. 1970;26(3):289-299.

33. Carroll CL, Goodman DM, Superina RA, et al. Timed Pediatric Risk of Mortality Scores predict outcomes in pediatric liver transplant recipients. Pediatr Transplant. 2003;7(4):289-295.

34. Chan PC, Chen HL, Ni YH, et al. Outcome predictors of fulminant hepatic failure in children. J Formos Med Assoc. 2004;103(6):432-436. 